\begin{tabular}{c|c|c}
\hline \hline & CLIMATE RESEARCH & Published April 19 \\
Vol. 26: $77-84,2004$ & Clim Res & \\
\hline \hline
\end{tabular}

\title{
Effect of ancient Inuit fur parka ruffs on facial heat transfer
}

\author{
Aline J. Cotel ${ }^{1, *}$, Raymond Golingo ${ }^{2}$, Jill E. Oakes ${ }^{3}$, Rick R. Riewe ${ }^{4}$ \\ ${ }^{1}$ Department of Civil and Environmental Engineering, University of Michigan, Ann Arbor, Michigan 48109-2125, USA \\ ${ }^{2}$ Department of Aeronautics and Astronautics, University of Washington, Seattle, Washington 98195-2400, USA \\ ${ }^{3}$ Department of Native Studies, and ${ }^{4}$ Department of Zoology, University of Manitoba, Winnipeg, Manitoba R3T 2N2, Canada
}

\begin{abstract}
The traditional clothing system developed and used by the Inuit is the most effective cold weather clothing developed to date. One of the key elements used by the Inuit is a fur ruff attached to the hood, hem, and cuffs of their parkas. This paper determines why the fur ruff is so critical to the effectiveness of cold-weather clothing, especially in protecting the face, without impeding movement or view, so essential to the Inuit hunter. To quantify the effectiveness of this clothing, heat transfer was measured on a model placed in a subsonic wind tunnel. The wind velocity and angle to the wind were varied. A boundary layer forms on the face, and heat transfer was measured across that layer using thermocouples. Different fur ruff geometries were examined to determine which was most efficient. The experimental results were combined with data collected using ethno-historical methods since 1970 by 2 of the authors. The traditional headgear proved to be the most efficient. The lowest heat transfer was found for the sunburst fur ruff geometry at different angles of attack and wind speeds. This unique combination of scientific and traditional Aboriginal knowledge provides a holistic perspective on new insights into the effectiveness of cold-weather clothing systems.
\end{abstract}

KEY WORDS: Cold-weather clothing $\cdot$ Inuit fur ruff design $\cdot$ Heat transfer $\cdot$ Wind-tunnel measurements

\section{INTRODUCTION}

Native peoples have flourished in the extreme cold of the Arctic for millennia. Arctic people living throughout the circumpolar region have time-tested their caribou (or reindeer) skin clothing ensembles for 3000 to 8000 yr (Stefansson 1944, 1955). During that time, their clothing has evolved, so that today they are able to live comfortably within the region, in spite of the bitter weather (Oakes \& Riewe 1995, 1998). Caribou, seal, and other animal skins provide adequate insulation for the covered regions of their bodies. This insulation is, in many respects, superior to modern synthetic materials, a subject discussed previously (Oakes et al. 1995). Perhaps even more remarkable is the performance of the headgear in protecting the exposed part of the body, the face. The Greenlandic Inuhuit, the Canadian Inuit, and the Inupiat and the Alaskan Inupiat and Yup'ik usually wear a parka style which has an attached hood with a fur ruff to protect the face. Many of the northern Siberian peoples, including the Saami, Nenets, Khanty,
Evenki, Nganasan, Chukchi and Siberian Yupik wear reindeer skin parkas, some with attached hoods and others with detachable hoods. During the winter these herders and hunters wear 1 or 2 inner parkas, plus an outer parka. Often, either an inner parka or the outer parka has an attached hood. These hoods are usually trimmed with an Arctic fox tail, or a broad strip of dog, wolf or wolverine fur. The Ngansan, living on the Tymir Peninsula, often wear a broad parka ruff of dog or wolverine fur, very similar to the Western Canadian Inuit or Alaskan Inupiat (Oakes \& Riewe 1998).

The Inuit living on the west side of Hudson Bay are an exception to this generality, in that they do not usually wear outside ruffs on their hoods; instead they sew a carefully cut caribou fur facing onto the inside edge of the hood. The facing and hood pattern are laid out so that the hair protrudes about $2 \mathrm{~cm}$ or so past the face, thereby breaking the wind. There are other examples of Inuit wearing impractical designs, such as the Copper Inuit with their pre-1914 parkas, which lacked a fur ruff and exposed the wrists to frostbite (Jenness 1946). 
The face must be exposed in order to see for hunting or travel. It is therefore essential to minimize facial heat transfer for frostbite prevention and survival. Not only does the Inuit parka ruff, which is worn behind the cheek bones, allow the wearer to see while hunting and traveling, it also drastically reduces the amount of hoar frost that otherwise builds up around a hood if worn in front of the cheek bones (as is the case with southern-style hoods). This frost build-up impairs vision and acts as a conductor, drawing heat from the face, thereby increasing the possibility of frostbite.

Arctic environments are very susceptible to high winds and low temperatures, which are ideal conditions for frostbite. Tissue freezes at or below a skin temperature of $-4^{\circ} \mathrm{C}$. The National Oceanic and Atmospheric Administration (NOAA) has developed wind chill tables to allow for a rapid assessment of frostbite danger. For example, at an air temperature of $-20^{\circ} \mathrm{C}$ with wind speed of $45 \mathrm{~km} \mathrm{~h}^{-1}$ at $10 \mathrm{~m}$ altitude, the wind chill is $-42^{\circ} \mathrm{C}$, and frostbite will occur after $30 \mathrm{~min}$ of exposure (Osczevski 1995, Bluestein \& Zecher 1999). People living in arctic conditions have developed means of protecting themselves against frostbite and hypothermia.

In particular, how can the face be protected from frostbite in such an environment, in the absence of modern technology? The question can be rephrased: is the traditional headgear optimal for protecting the face?

Caribou Inuit elders recommend using hoods that fit loosely around the back of the head and closely at the face (Oakes \& Riewe 1995). The space behind the head allows for warmer air rising from the torso region. In addition, the fur used is usually wolverine, wolf or dog. Inuit hunters have observed that irregular hair lengths help break the wind and therefore create a calm micro-environment around the face. One of the goals of this paper is to relate those observations to engineering concepts and technical parameters that can be tested and evaluated in a laboratory environment.

The effectiveness of Inuit clothing is estimated by quantifying heat transfer from the skin to the surrounding air. Heat transfer always occurs across a boundary layer, a relatively thin layer of nearly stagnant air adjacent to the skin. In general, the heat transfer is inversely proportional to the thickness of the boundary layer. Consequently, a thin boundary layer implies relatively rapid cooling for a given temperature difference across the layer. The behavior of the boundary layer is key to understanding the facial heat loss.

Some furs, particularly wolverine, arctic wolf, husky dog, coyote, and skunk, have long stiff guard hairs and dense under fur. Wolverine fur has been proven to be superior to all other natural furs used as parka ruffs (Mizell et al. 1956). Wolverine fur's superior qualities include the protection it provides from the wind, the ease with which it sheds ice and frost, the comfort it provides the face, and its durability. These attributes are due to a complex combination of structural and mechanical characteristics including the fur's diameter, length, and hair spacing on the skin (Hardy 1948, Blouin \& Cowie 1949, US Naval Air Experimental Station 1949). Fur elements behave in a similar fashion to roughness elements on an airplane wing, for example, or dimples on a golf ball. The effect of roughness is to increase the boundary layer thickness. The Prandtl number (ratio of kinematic viscosity to thermal diffusivity) for air at a temperature ranging from -50 to $300^{\circ} \mathrm{C}$ is on the order of unity (Schlichting 1979). Therefore, the thickness of both the velocity and thermal boundary layers is approximately the same, and roughness elements will affect both boundary layers. As will be seen in more detail in the next section, the thickness of the boundary layer is inversely proportional to heat transfer. Introducing fur within the boundary-layer region is similar to adding roughness elements to the surface. It increases the thickness of the boundary layer, thereby reducing the heat-transfer coefficient where the boundary layer is attached.

In summary, it is evident that the fur ruff designs developed and worn in the Arctic over several thousand years have been optimized to protect the face from the extreme cold. This paper attempts to quantify the effectiveness of such clothing by using a framework based on boundary-layer theory and by measuring heat transfer for different configurations: no hood, a fabric hood, a military hood and the sunburst fur ruff.

\section{BOUNDARY-LAYER BEHAVIOR}

Frictional forces slow the movement of fluid near the surface of any object placed in a flow (Schlichting 1979). These effects are confined to a thin layer close to the surface; within this thin layer, the velocity varies from zero at the wall to the full free-stream velocity right outside the layer. This concept was developed by Prandtl (1905), and the layer is called the boundary layer. A characteristic of boundary-layer behavior is boundary-layer separation. This occurs when the fluid particles from the boundary layer are forced outwards. This creates a reverse flow within the boundary layer, and vortices are formed during that process. This phenomenon is most common for bluff bodies, such as circular cylinders and spheres (Fig. 1). The boundary layer is attached to the cylinder surface on the upstream face of the cylinder, but is unable to resist the adverse pressure gradient close to the top and bottom locations of the cylinder (Crowe et al. 2001). At those locations, the boundary layer separates from the cylinder surface and vortices are formed. The location of vortex sheet roll-up downstream of the separation 


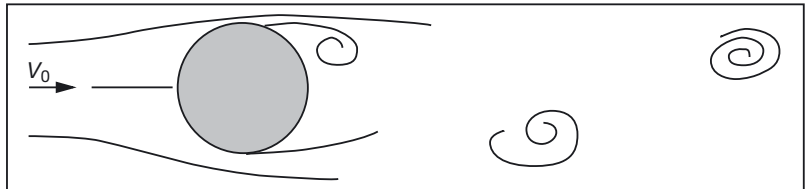

Fig. 1. Flow over a cylinder (Crowe et al. 2001). Note the region of separation behind the cylinder and the presence of eddies in the flow

point is proportional to the boundary-layer thickness at the separation point. Once vortices are formed, they will increase heat transfer, as they move fluid (here warmer air) from the surface of the cylinder towards the surrounding environment (colder air). Therefore, the location of the vortex sheet roll-up is important in understanding and quantifying heat transfer.

If the surface is not smooth, an additional parameter comes in: surface roughness. Depending on the size of the roughness elements, the boundary layer will be affected. Roughness will trigger a transition from a laminar to a turbulent boundary layer further upstream than in the smooth case, and it will delay separation of the boundary layer. Here, fur of different hair lengths (called broad- or wide-spectrum fur) acts as roughness elements for the boundary layer. This contributes to a thickening of the boundary layer and a delay of the vortex sheet roll-up, further reducing the heat transfer.

If all the hair fibers in the fur were of the same length, such as arctic hare fur, then the momentum thickness of the boundary layer would be much less than for a wide spectrum of fiber lengths. For a narrow spectrum of fiber lengths, when the boundary layer separates at the edge of the fur ruff, the relatively thin vortex sheet would quickly roll up into discrete vortices. The turbulent shear layer would promptly reattach, with a relatively high heat-transfer rate. So a wide spectrum of fiber length presumably plays an important role in reducing heat transfer.

The temperature field around a hot body in a fluid stream is of the boundary-layer type. The temperature field is contained within a thin layer close to the surface. This thin layer is a region of steep temperature gradients. There, the heat flux due to conduction is of the same order of magnitude as that due to convection. Radiative heat transfer can be ignored in these conditions. As pointed out by Schlichting (1979), radiative heat transfer is usually a small component of the total heat transfer for bodies in flowing fluid. Using the findings of Zemansky (1957), the radiative component was calculated using the specific data of our experiments. The total heat transfer was measured to be on the order of $1000 \mathrm{~W} \mathrm{~m}^{-2}$ in this study.

As an idealization of the more complicated shape of the human head, consider the flow past a sphere or cir- cular cylinder of diameter $d$. The flow approaches this bluff body smoothly with free-stream wind of speed $U$. One streamline brings fluid to rest at the stagnation point on the windward side of the body. In the vicinity of the stagnation point, the boundary layer has a constant thickness:

$$
\delta \approx \sqrt{\frac{d}{U} v}
$$

where $v$ is the kinematic viscosity of air, approximately $0.148 \mathrm{~cm}^{2} \mathrm{~s}^{-1}$ for an air temperature of $20^{\circ} \mathrm{C}, d$ the cylinder diameter and $U$ the free-stream velocity. The boundary layer thickness $\delta$ is proportional to the square root of the diameter. If the face were pointed directly into the wind, at least part of it would be in this stagnation region.

The dramatic sunburst fur ruff geometry (Fig. 2) increases the stagnation region boundary layer thickness over the face by increasing the diameter of the bluff body and by placing the face closer to the stagnation point with respect to the diameter.

If the face is turned slightly away from the wind, the stagnation point is no longer at the center of the face, and the boundary layer flows over the face. Suppose the head is covered by the hood of a parka, and the hood is edged in fur. An idealized geometry for the local flow from the fur to the face is the flow over a backward-

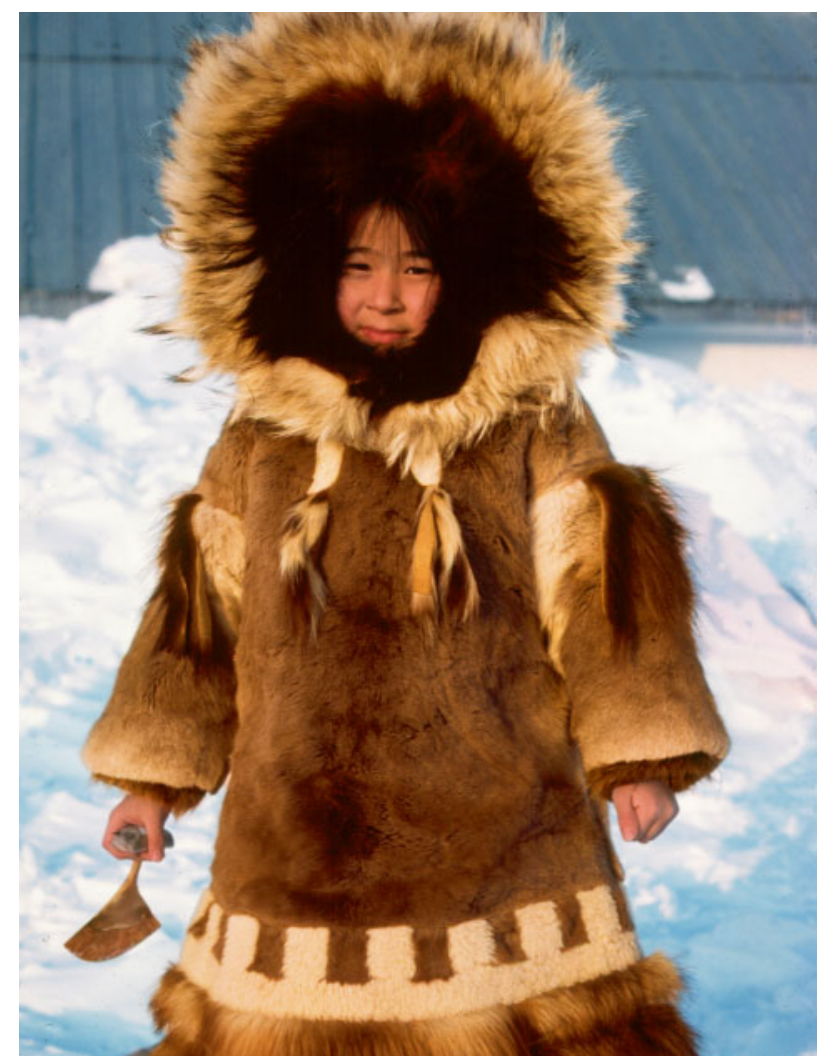

Fig. 2. Example of a fur ruff-sunburst configuration 
$\stackrel{\text { Flow direction }}{\longrightarrow}$

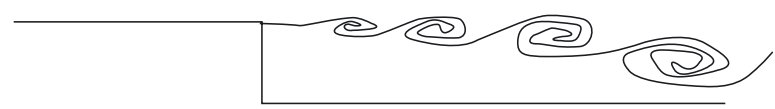

Fig. 3. Backward-facing step-flow schematic. A shear layer is created between the re-circulating region behind the stepregion fluid and the rest of the flow. Flow direction is from left to right

facing step (Fig. 3). The backward-facing step represents the difference in height between the ruff and the face, as the ruff has a certain thickness. The boundary layer, composed of a vortex sheet, separates at the lip of the step, forming a free vortex sheet or shear layer. The vortex sheet rolls up into discrete vortices, at a downstream station proportional to the initial vortex-sheet thickness at the separation point of the boundary layer. The discrete vortices have a large entrainment appetite. This draws the layer toward the surface, causing it to reattach at some downstream station. In the vicinity of the reattachment, the heat-transfer coefficient is increased by the vigorous turbulent transport from the discrete vortices near the surface. There are 2 important length scales in this problem, the thickness of the separating boundary layer and the height of the backward-facing step. They determine a third length scale, the location of downstream reattachment and the heat transfer from the surface.

a
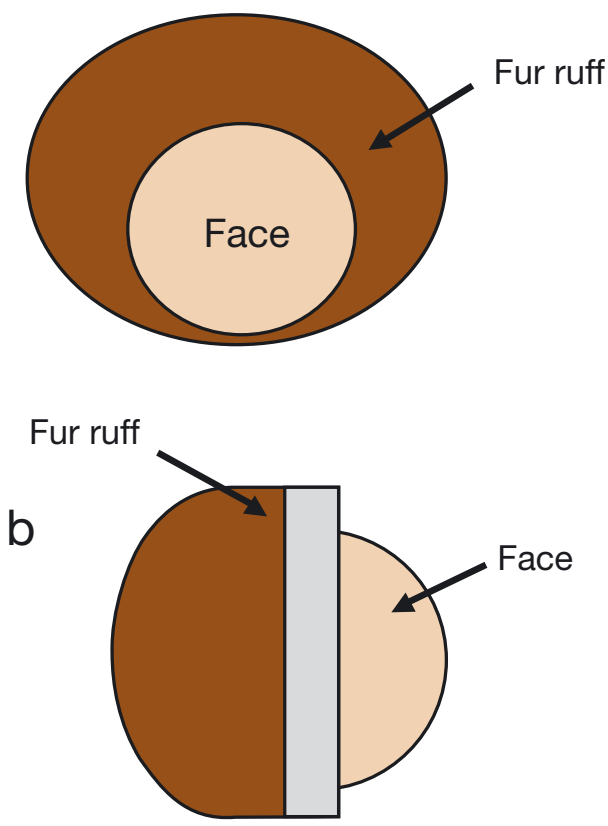

Fig. 4. Schematic of the fur ruff geometry representing the size and location of the sunburst fur ruff with respect to a human face: (a) front view and (b) side view
Fur ruff designs, such as the sunburst geometry, that combine a large diameter and the presence of broadspectrum fur increase the boundary layer thickness, therefore favorably reducing the heat transfer across the boundary layer. These hypotheses are quantified using heat-transfer measurements on a model at different angles of incidence and wind speeds, for a number of geometries.

\section{EXPERIMENT}

\subsection{Experimental facility}

The experiments were undertaken in the Low-Speed Wind Tunnel in the Department of Aeronautics and Astronautics at University of Washington in Seattle. The wind tunnel is an open-circuit model, with a $1 \mathrm{~m} \times$ $1 \mathrm{~m}$ cross-section that is $2.7 \mathrm{~m}$ long. It is ideal for testing models up to speeds of $220 \mathrm{~km} \mathrm{~h}^{-1}$. The windtunnel temperature was around $23-24^{\circ} \mathrm{C}$. It was measured for each test, as it might have varied by 1 or $2^{\circ} \mathrm{C}$, depending on the test day.

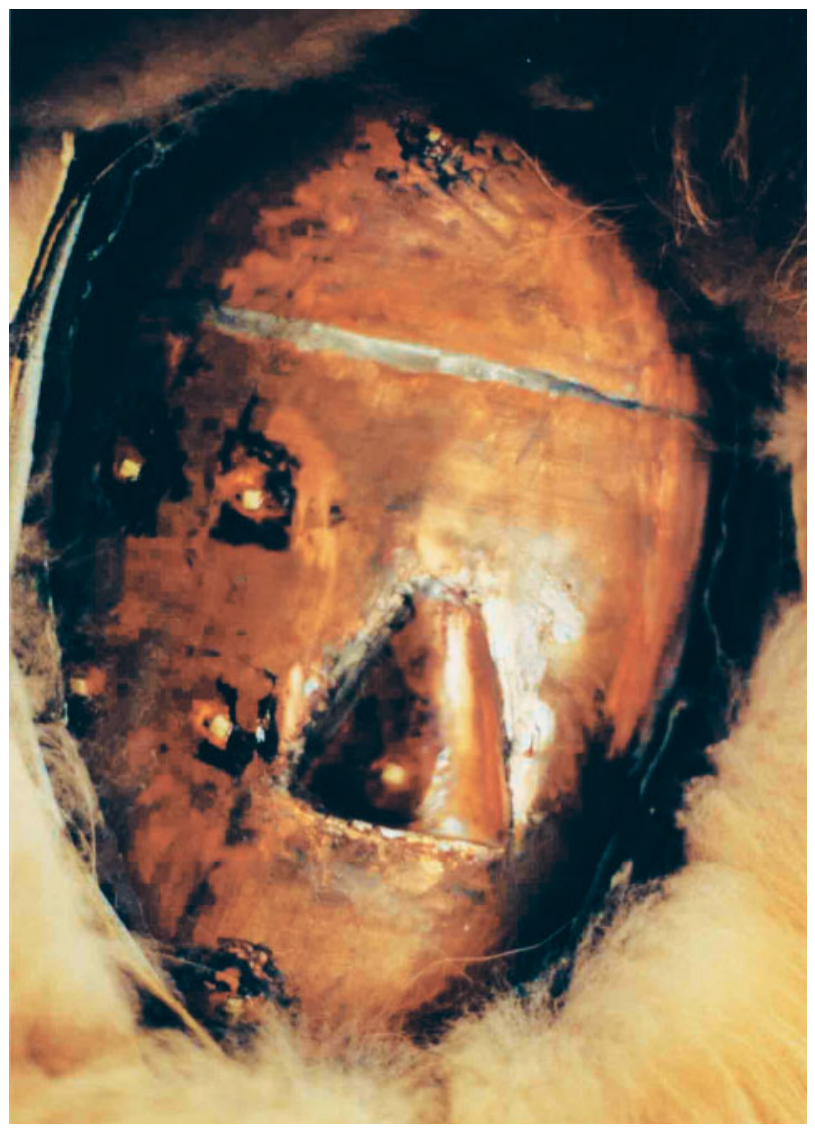

Fig. 5. Copper model with thermocouples. The white areas (Teflon) represent the location of the thermocouples used to take the heat-transfer measurements 
An idealized model of the face was built, using the combination of a cylinder and half-sphere (Figs. 4 \& 5). The model has an internal electrical heating element to provide a temperature difference between it and the air in the wind tunnel. The strip heater $(20 \mathrm{~cm}$ in length, $120 \mathrm{~V}$ ) is mounted in the center of the head to heat it to $100^{\circ} \mathrm{C}$. The model is constructed of copper, so that heat is readily conducted to the surface for a strong temperature flux signal.

Models of fur ruffs to fit the scaled-down model were made (Fig. 6). Different types of fur ruffs were tried, to determine the effect of hair of different lengths on the boundary layer and to find the optimum configuration. Fig. 7 compares the military hood geometry with the sunburst one.

\subsection{Experimental method}

The heat transfer across the boundary layer was measured at many locations on the sphere using 14

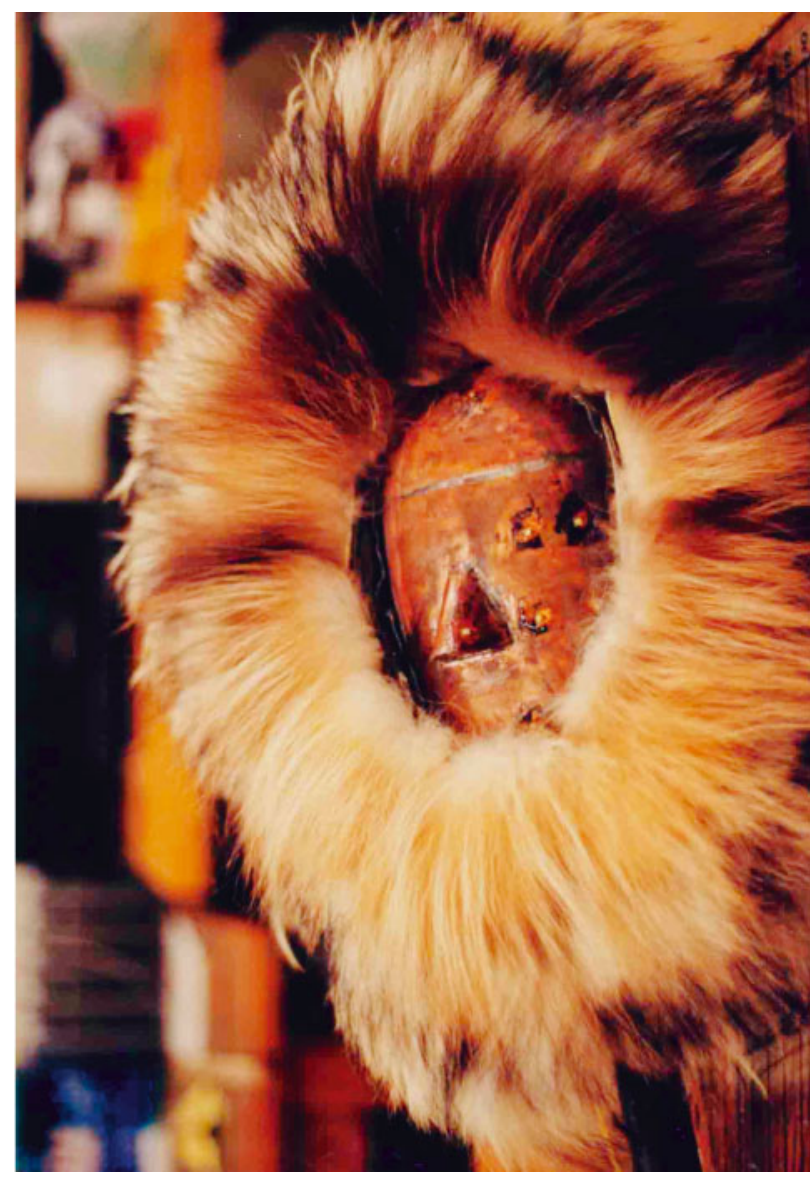

Fig. 6. Copper model with fur ruff. The ruff is positioned with respect to the copper cylinder. The thermocouples are also visible on the right side of the photograph, between the nose and the fur ruff
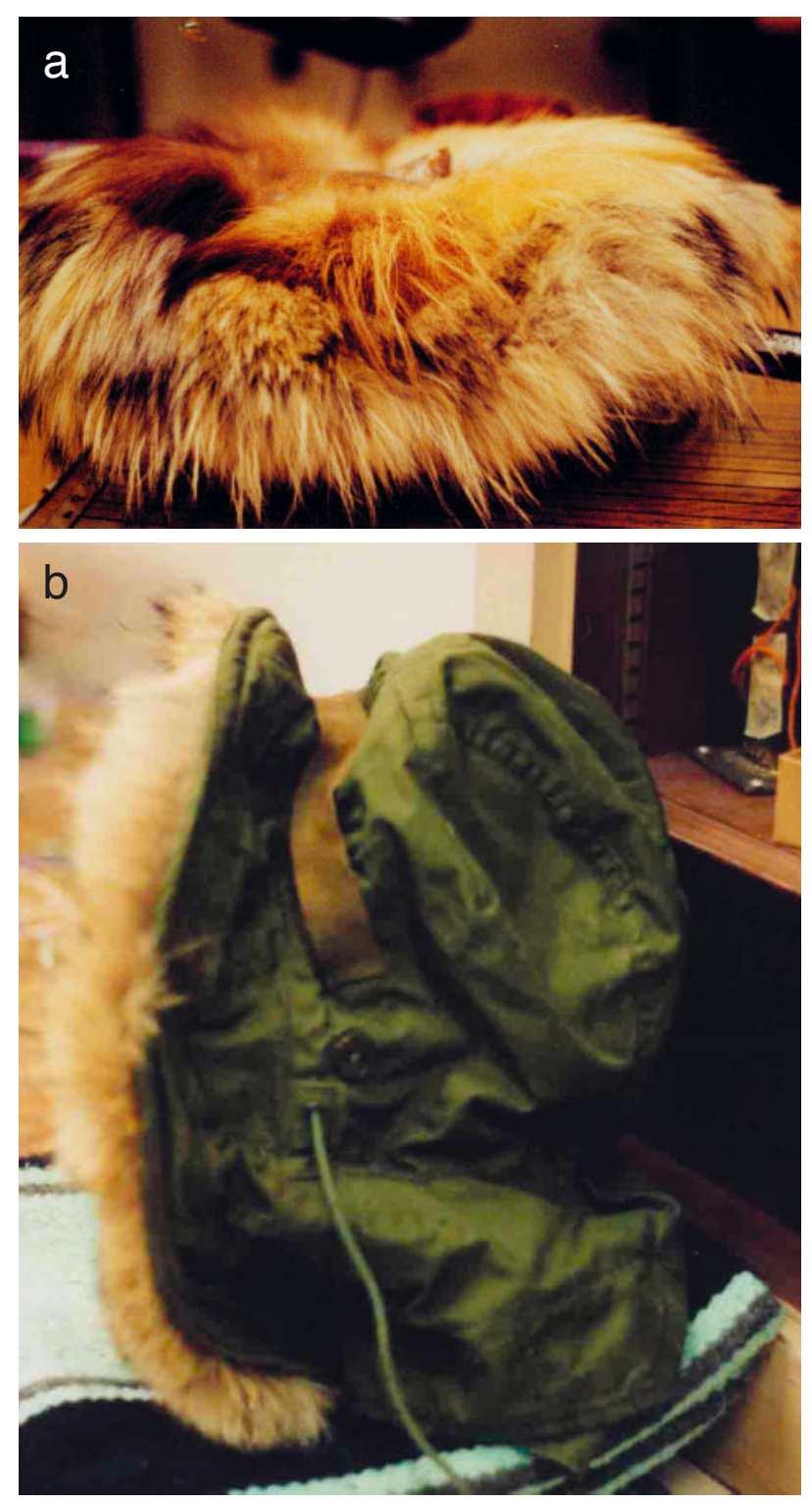

Fig. 7. Comparison between (a) sunburst and (b) military geometries. The difference in fur length and thickness is shown in the photographs

pairs of thermocouples (Fig. 8). The following calibration method was used. The thermocouples were connected to the electronics used in this particular set-up. They were then placed in a beaker with water and melting ice $\left(0^{\circ} \mathrm{C}\right)$. The temperature readings from each thermocouple were then taken. The water was brought to a boil $\left(100^{\circ} \mathrm{C}\right)$, and the barometric pressure was recorded. The temperature readings were again recorded. A straight line was fitted between the points, giving the relationship between the read temperature and actual temperature. The temperature gauges were placed at the surface, one on top of the other, with a thin layer of Teflon separating them. The surface 


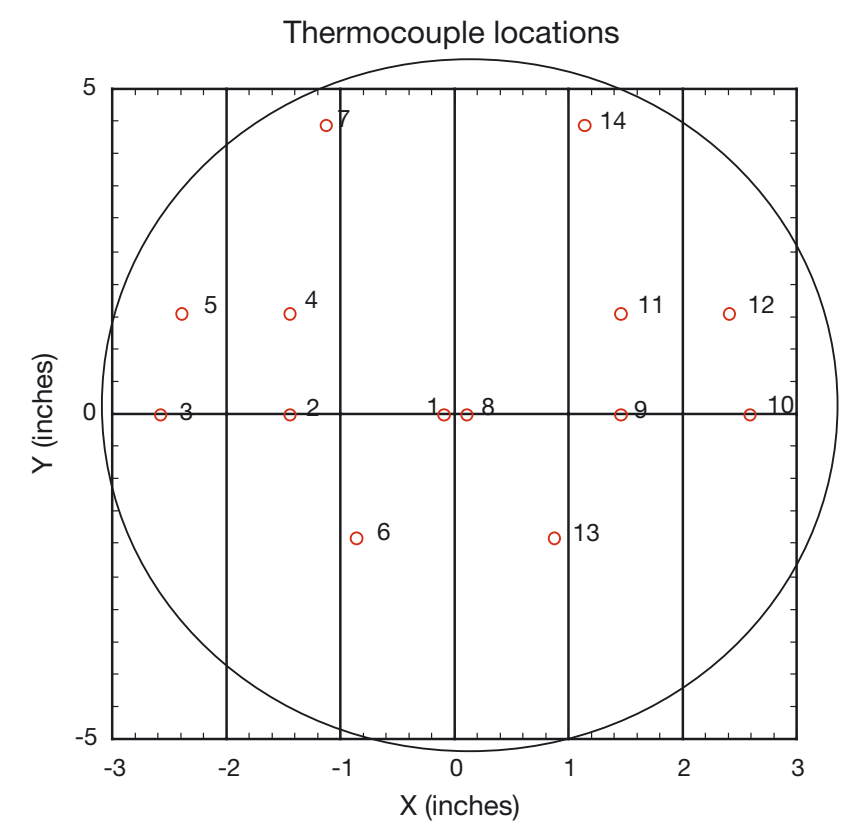

\begin{tabular}{|ccc|}
\hline Thermocouple & $x$ (inches) & $y$ (inches) \\
\hline 1 & -0.1 & 0 \\
2 & -1.45 & 0 \\
3 & -2.58 & 0 \\
4 & -1.45 & 1.55 \\
5 & -2.4 & 1.55 \\
6 & -0.861 & -1.9 \\
7 & -1.14 & 4.45 \\
8 & 0.1 & 0 \\
9 & 1.45 & 0 \\
10 & 2.58 & 0 \\
11 & 1.45 & 1.55 \\
12 & 2.4 & 1.55 \\
13 & 0.861 & -1.9 \\
14 & 1.14 & 4.45 \\
\hline
\end{tabular}

Fig. 8. Schematic of thermocouple locations. Red circles represent the location of the thermocouples. The table gives each thermocouple's location in inches in the $x$ and $y$ directions. One inch $=2.5 \mathrm{~cm}$

heat flux was determined by measuring the temperature difference across the conducting Teflon layer. This temperature difference divided by the thickness of the conducting layer was approximately the temperature gradient. The temperature gradient times the thermal conductivity of the conducting layer gave the surface heat transfer.

For each geometry of fur ruffs, the wind speed and the angle of incidence (or yaw angle) were varied. The heat transfer was measured for each condition at all 14 locations. The yaw angle was varied from 0 to $90^{\circ}$. No tests were performed at $180^{\circ}$ because the flow is fully separated and the differences between headgear geometry are expected to be small and uninteresting. The critical situation of a high rate of heat loss is when the head faces into the wind.
Two dimensionless parameters were chosen to describe the results. Because of the non-dimensionless nature of these numbers, temperature and viscosity in the laboratory facility do not have to be perfectly equal to those in the Arctic. Instead, the Reynolds number $(\mathrm{Re})$ is used to match the environment's conditions.

The flow parameter used was the dimensionless Reynolds number:

$$
\operatorname{Re}=\frac{U d}{v}
$$

where $U$ is the wind speed $\left(\mathrm{m} \mathrm{s}^{-1}\right), d$ the sphere diameter $(\mathrm{m})$ and $v$ the kinematic viscosity of air $\left(\mathrm{m}^{2} \mathrm{~s}^{-1}\right)$. It describes the ratio of inertial to viscous forces and determines if a flow is turbulent or laminar. By changing the wind speed, Re was varied by a factor of 3 . The air viscosity at $20^{\circ} \mathrm{C}$ is $14.8 \times 10^{-6} \mathrm{~m}^{2} \mathrm{~s}^{-1}$. At $-20^{\circ} \mathrm{C}$, the air viscosity drops to a value of $11.2 \times 10^{-6} \mathrm{~m}^{2} \mathrm{~s}^{-1}$. In our experiments, we chose to vary Re and not the temperature and viscosity of air. The change of viscosity between 20 and $-20^{\circ} \mathrm{C}$ is on the order of $24 \%$; however, the range of Re was far greater than the change in viscosity. In addition, the viscosity is not important once the flow separates. Its main role is in setting the initial boundary layer thickness at the separation point.

The Nusselt number $(\mathrm{Nu})$ was used to quantify the heat transfer. A dimensionless number, it is the ratio of actual heat transfer to characteristic laminar heat-transfer value:

$$
\mathrm{Nu}=\frac{h d}{k_{\text {air }}}
$$

where $h$ is the heat-transfer coefficient $\left(\mathrm{W} \mathrm{K}^{-1} \mathrm{~m}^{-2}\right), d$ is the diameter of the sphere $(0.15 \mathrm{~m})$ and $k_{\text {air }}$ is the thermal conductivity of the air $\left(0.0263 \mathrm{~W} \mathrm{~K}^{-1} \mathrm{~m}^{-1}\right)$.

In turn, the heat transfer coefficient is defined as:

$$
h=\frac{k_{\text {Teflon }} \Delta T}{L\left(T_{\text {surface }}-T_{\text {tunnel }}\right)}
$$

where $k_{\text {Teflon }}$ is the thermal conductivity of Teflon $\left(0.25 \mathrm{~W} \mathrm{~K}^{-1} \mathrm{~m}^{-1}\right), L$ is the thickness of the Teflon layer $(1 \mathrm{~mm})$ and $\Delta T$ is the temperature difference across the Teflon material.

What matters in heat-transfer measurements and for Nusselt numbers is the magnitude of the temperature difference, not its sign, as long as buoyancy can be neglected. In the present experiments, the wind-tunnel temperature was kept at ambient temperature, and the model was heated to achieve temperature differences similar to those encountered in arctic climates.

Fourteen thermocouples were placed on the surface of the model (Fig. 8). For each thermocouple location, the heat-transfer coefficient and Nusselt number were calculated based on the above equations. Finally, an average of the Nusselt numbers over the entire face was taken for each angle of incidence and Re. 


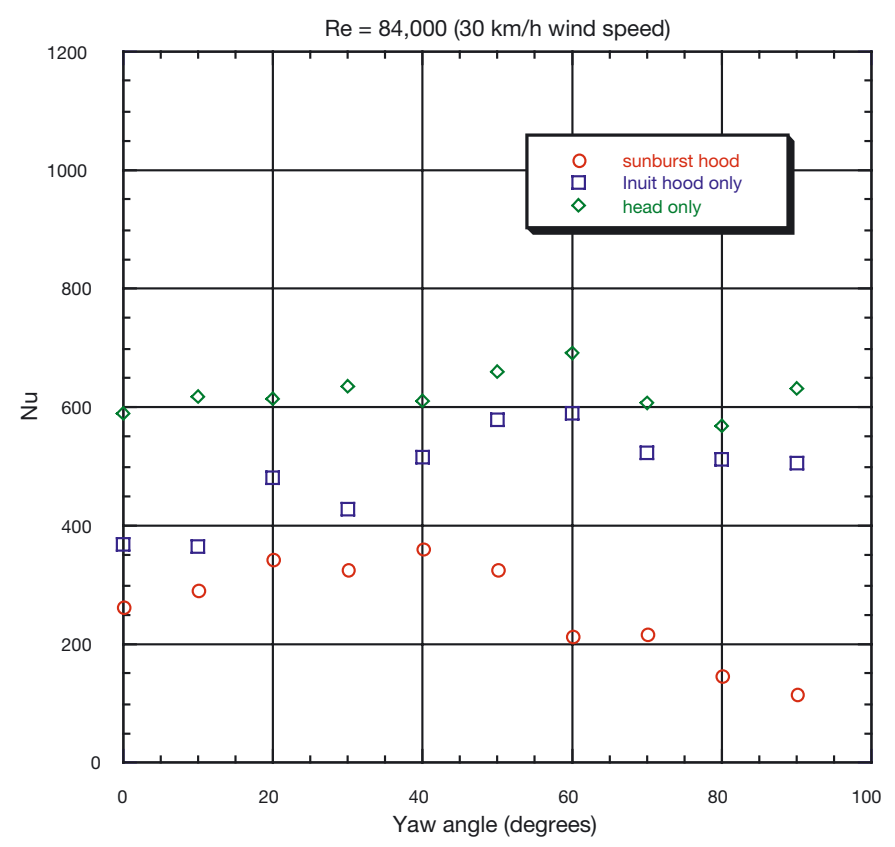

Fig. 9. Heat-transfer data for $30 \mathrm{~km} \mathrm{~h}^{-1}$ wind. The Reynolds number $(\mathrm{Re})$ is held constant, and heat transfer is measured as a function of Nusselt number $(\mathrm{Nu})$ based on the head diameter for different geometries and for different angles of attack

\section{RESULTS}

The heat-transfer results are shown in Figs. 9-11. They present the dimensionless heat-transfer coefficient or Nusselt number as a function of the yaw angle for several configurations. The yaw angle is defined as the angle between the nose of the model and the free-stream direction. At $\mathrm{Re}=84000$, corresponding to a wind speed of $30 \mathrm{~km} \mathrm{~h}^{-1}$, for all yaw angles, the sunburst fur ruff was by far the most efficient. This was particularly true at high yaw angles (Fig. 9). The sunburst fur ruff reduced the heat transfer by as much as a factor of 6 . The sunburst design was also more sensitive to yaw angle. For this wind speed, the lowest heat transfer was measured at large yaw angles with the sunburst configuration. The highest heat transfer was measured for the head-only geometry. Adding the hood (without fur) decreased the heat transfer only slightly. The addition of the sunburst fur ruff to the hood drastically reduced the heat transfer.

This pattern was also observed at a higher value of $\operatorname{Re}=166000$, corresponding to a wind speed of $60 \mathrm{~km}$ $\mathrm{h}^{-1}$ (Fig. 10). In addition to the 3 configurations previously tested for $30 \mathrm{~km} \mathrm{~h}^{-1}$, a military hood with a fur ruff was fitted on the copper head. The military hood is composed of uniform and short hair compared to the sunburst fur ruff. For the 3 yaw angles tested, the military geometry was inferior to the sunburst design in terms of minimizing the heat transfer. At large yaw angles, the heat transfer for the sunburst was 3 times lower than for the military hood's ruff. It was close to a factor of 2 at low yaw angles.

For a wind velocity of $90 \mathrm{~km} \mathrm{~h}^{-1}(\mathrm{Re}=246000)$, only 2 geometries were tested (Fig. 11). There was a greater amount of scatter for these tests. It was difficult for some yaw angles to keep the hood stationary due to the large velocity and highly turbulent nature of the flow. At most yaw angles, the sunburst geometry exhibited the least amount of heat transfer.

The sunburst geometry provided the least amount of heat transfer for the range of Re and yaw angles tested, when compared to the other types of geometries, i.e. head alone, hood only and military hood.

In addition, the hypothesis of wide-spectrum fur being beneficial in reducing the heat transfer proved to be correct as far as the present measurements show. The military hood used in the tests had short hair of uniform length. The sunburst fur ruff has different lengths of hair, i.e. a wider spectrum of fur. As predicted, the heat transfer for the military type hood was greater than that of the sunburst fur ruff.

The hypotheses that the thickness of the boundary layer and the type of fur used are important parameters were verified by the present measurements. By optimizing the boundary-layer behavior, the heat transfer can be minimized.

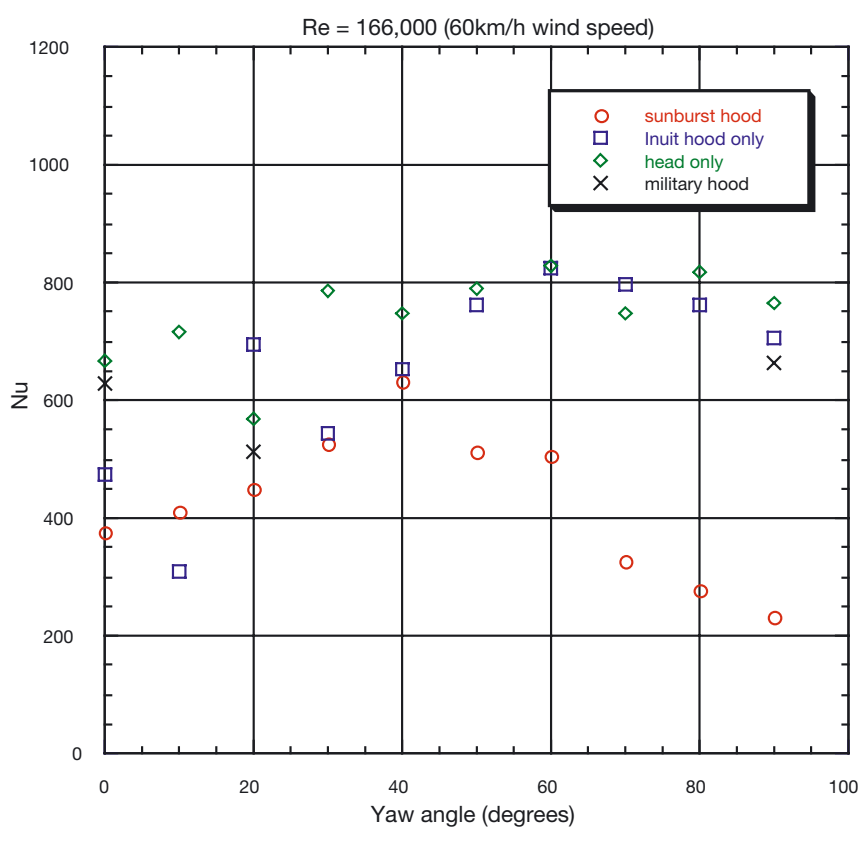

Fig. 10. Heat-transfer data for $60 \mathrm{~km} \mathrm{~h}^{-1}$ wind. Details as in Fig. 9 


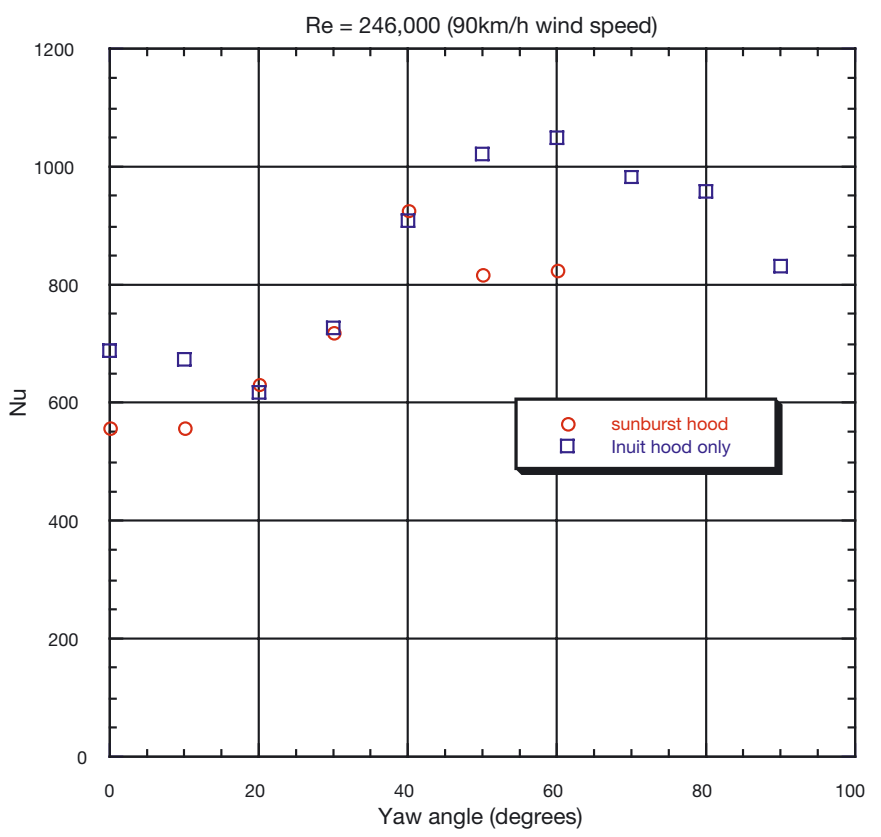

Fig. 11. Heat-transfer data for $90 \mathrm{~km} \mathrm{~h}^{-1}$ wind. Details as in Fig. 9

\section{CONCLUSION}

Experiments were performed in a wind tunnel where traditional Inuit headgear was tested for effectiveness in reducing heat transfer, and thereby preventing facial heat loss. Different geometries were tested and compared for the same range of Reynolds numbers and yaw angles. The working hypotheses based on the concept of boundary-layer thickness and separation characteristics were validated with heat-transfer measurements. Heat transfer was measured by averaging heat transfer at 14 locations on a model made of copper.

The present experiments clearly demonstrate the superiority of the sunburst fur ruff configuration for all wind velocities and yaw angles tested. Heat transfer is reduced due to the action of 2 parameters, the large diameter of the sunburst ruff and the wide-spectrum fur on the headgear. Both parameters tend to increase the boundary-layer thickness, therefore yielding lower heat transfer. This geometry proved remarkably effective, as the heat transfer can be lowered by a factor of 6 for a wind speed of $30 \mathrm{~km} \mathrm{~h}^{-1}$, when compared with the head-only configuration. A reduction of a factor of 4 was observed at $60 \mathrm{~km} \mathrm{~h}^{-1}$.

The superior effectiveness of this piece of clothing has been known by Inuit hunters and seamstresses, who have thrived for thousands of years by creating polar ruff designs that provide protection against the cold, windy arctic climates; it is quantified for the first time in this paper. The sunburst fur ruff design is truly a remarkable 'time-tested' design. It is somewhat perplexing, however, that the Central Canadian Arctic Inuit and the pre-1914 Copper Inuit did not wear external ruffs, despite living in one of the coldest, windiest regions of North America. For over 30 yr, R. R. Riewe and J. E. Oakes have worn parka styles with and without external ruffs and found a vast difference in comfort level. Fashion and tradition is very important to the Inuit (Oakes 1986, 1991). The lack of a parka ruff may be a fashion statement or tradition-one that is far less comfortable for the wearer.

\section{LITERATURE CITED}

Blouin AE, Cowie WE (1949) Investigations of the frosting of wolverine fur. Report No. 10, Defence Research Chemical Laboratories, Ottawa

Bluestein M, Zecher J (1999) A new approach to an accurate wind chill factor. Bull Am Meteorol Soc 80:1893-1900

Crowe CT, Roberson JA, Elger DF (2001) Engineering fluid mechanics, 7th edn. Wiley, New York

Hardy TMP (1948) Wolverine fur frosting. J Wildl Manag 12: $331-332$

Jenness D (1946) Material culture of the Copper Eskimo. Report of the Canadian Arctic Expedition 1913-1918. Vol. XVI. Southern Party 1913-1916. Kings Printer, Ottawa

Mizell LR, Frishman D, Saxon R (1956) Development of non-critical substitutes for wolverine fur. Report No. 92, Textile Series, Headquarters Quartermaster Research and Development Command, Harris Research Laboratories, Washington, DC

Oakes J (1986) Inuit Annuraangit, our clothes. A travelling exhibition of Inuit clothing. Thumb Prints, Ottawa

Oakes J (1991) Copper and caribou Inuit skin clothing production. Mercury Series Paper No. 118, Canadian Ethnology Service, Canadian Museum of Civilization, Ottawa, p 14-101

Oakes J, Riewe R (1995) Our boots; an Inuit women's art. Douglas \& McIntyre, Vancouver

Oakes J, Riewe R (1998) Spirit of Siberia: traditional native life, clothing, and footwear. Douglas \& McIntyre, Vancouver, and Smithsonian Institution, Washington, DC

Oakes J, Wilkins H, Riewe R, Kelker D, Forest T (1995) Comparison of traditional and manufactured cold weather ensembles. Clim Res 5:83-90

Osczevski RJ (1995) The basis of wind chill. Arctic 48:372-382

Prandtl L (1905) Verhandlunger des dritten Internationalen Mathematiker-Kongresses in Heidelberg, 8-13 August 1904, p 484 (Translation in NASA Memo 452)

Schlichting H (1979) Boundary-layer theory. McGraw-Hill, New York, p 265-321

Stefansson V (1944) Arctic manual. Macmillan, New York

Stefansson V (1955) Clothes make the Eskimo. Natl Hist 64(1):32-41

US Naval Air Experimental Station (1949) Wolverine fur for use on arctic clothing - investigations of substitutes for. Report No. AML NAM AE 525060, Part I, Aeronautical Materials Laboratory, Philadelphia, PA

Zemansky MW (1957) Heat and thermodynamics. McGrawHill, New York, p 106 